\title{
ANÁLISE FAUNÍSTICA DOS PARASITÓIDES (HYMENOPTERA, BRACONIDAE) DE Anastrepha spp. (DIPTERA, TEPHRITIDAE) EM MANAUS E IRANDUBA, ESTADO DO AMAZONAS. ${ }^{1}$
}

\author{
Nelson A. D. CANAL ${ }^{2}$, Roberto A. ZUCCHP, Neliton M. SILVA', Sinval \\ SILVEIRA NETO ${ }^{3}$
}

\begin{abstract}
RESUMO - Foram coletados 2630 parasitóides de Anastrepha spp., pertencentes a cinco espécies de Braconidae, em quatro locais de dois municípios do Estado do Ámazonas. Opius sp. foi a espécie predominante no estudo, ocorrendo com maior freqüência na área urbana de Manaus. Doryctobracon areolatus (Szépligeti, 1911) foi a espécie predominante nas áreas rurais. As comunidades foram delimitadas e caracterizadas através de índices faunísticos. As comunidades apresentaram quocientes de similaridade entre $82 \mathrm{e} 100 \%$.
\end{abstract}

Palavras chaves: Ecologia, Índices faunísticos, Opiinae, Alysiinae, Moscas-das-frutas.

Faunistic Analysis of Parasitoids (Hymenoptera, Braconidae) of Anastrepha spp. (Diptera, Tephritidae) in Manaus and Iranduba, State of Amazonas, Brazil.

ABSTRACT - A total of 2,630 braconids from Anastrepha spp. were recovered in four locations of two counties (Manaus and Iranduba) in Amazonas State, Brazil. The parasitoids belong to five species. Opius sp. was the predominant species, showing the highest frequency in the downtown region of Manaus. On the other hand, Doryctobracon areolatus (Szépligeti, 1911) was the predominant species in the countryside. For the faunistic analysis, each collecting site was considered a community. The coefficient of similarity between communities varied from 82 to $100 \%$.

Key words: Ecology, Faunistic index, Opiinae, Alysinae, Fruit flies.

\section{INTRODUÇÃO}

As moscas-das-frutas (Diptera, Tephritidae) constituem um importante grupo de pragas de frutíferas e hortaliças no mundo todo. Tradicionalmente, têm sido controladas por métodos químico e cultural (CLAUSEN, 1956; WHARTON, 1989), entretanto, o controle biológico é tido atualmente como uma alternativa excelente para aumentar a eficiência do manejo integrado destas pragas (WHARTON, 1989).
Cerca de 82 espécies de parasitóides, pertencentes a várias famílias, têm sido obtidas de moscasdas-frutas, porém, a maioria delas e as mais importantes pertencem à família Braconidae, principalmente à subfamília Opiinae (WHARTON, 1989). A família Braconidae compreende aproximadamente 15000 espécies conhecidas, sendo a maior parte delas parasitóides de outros insetos (WHARTON, 1993). Dentro desta família, Opiinae e Alysiinae formam um grupo

Parte da dissertação apresentada à ESALQ/USP.

2 Universidad del Tolima (A.A. 549, Ibagué, Tol. Colômbia).

Aluno do curso de pós-graduação em Entomologia ESALQ/USP.

3 Departamento de Entomologia ESALQ/USP. Caixa Postal 9,

13418-900 Piracicaba, SP.

4 Faculdade de Ciências Agrárias/Universidade do Amazonas/Campus Universitário 69077-000 Manaus-AM. 
monofilético caracterizado pelo parasitismo de dípteros ciclorrafos, desenvolvendo-se como parasitóides solitários e coinobiontes, que empupam no interior do pupário do hospedeiro (GAULD \& BOLTON, 1988).

Os trabalhos com parasitóides de moscas-das-frutas iniciaram-se em 1902. Os casos de sucesso têm sido relatados em alguns países para o controle de várias espécies de tefritídeos (WHARTON, 1989), todavia, muitos programas de controle biológico não têm tido os resultados esperados, por problemas no transporte e na criação dos inimigos naturais, inadequada seleção da espécie de parasitóide e à falta de estudos no habitat nativo dos parasitóides (CLAUSEN, 1956; WHARTON, 1989).

A análise dos componentes da população são importantes meios para estabelecer os processos de predação e parasitismo. É necessário quantificar o grau de associação entre duas espécies para determinar, entre outros parâmetros, a relação predador-presa, além de estabelecer o passo inicial para a delimitação de uma comunidade (SOUTHWOOD, 1966).

Neste trabalho objetivou-se caracterizar quatro locais do Estado do Amazonas, em relação aos braconídeos parasitóides de moscasdas-frutas. Foram utilizados índices faunísticos para conhecer a interação localidade-parasitóide, assim como obter informações a respeito do comportamento dos parasitóides nas comunidades estudadas.

\section{Material e Métodos}

\section{Coleta dos parasitóides.}

Os parasitóides foram coletados durante levantamentos populacionais de moscas-das-frutas. Foram amostradas 35 espécies de frutíferas nativas e exóticas, hospedeiras convencionais e potenciais de moscasdas-frutas. Frutos, com sintomas de ataque por moscas-das-frutas, foram coletados e mantidos no laboratório em caixas contendo areia umedecida. Uma semana após, obtinham-se por peneiramento as pupas, que eram transferidas para frascos de vidro até a emergência da mosca ou do parasitóide (SILVA, 1993). A associação mosca/parasitóide só foi considerada quando emergiu apenas uma espécie de mosca-das-frutas e do parasitóide, provenientes de determinado fruto, no tubo de criação.

\section{Locais de coleta.}

Os parasitóides foram coletados em quatro locais do Estado do Amazonas, nos municípios de Iranduba e de Manaus. No primeiro, as coletas foram feitas em pomares não comerciais e frutíferas silvestres da área rural do município; em Manaus as coletas realizaram-se em três locais: área urbana, Estação Experimental de Frutíferas Tropicais do Instituto Nacional de Pesquisas da Amazônia, INPA (rodovia BR174, km 40) e no bairro distrital do Aleixo (Fig. 1). O clima da região é do tipo "Afi" (quente e úmido), pela classificação de Köppen com precipitação média anual 


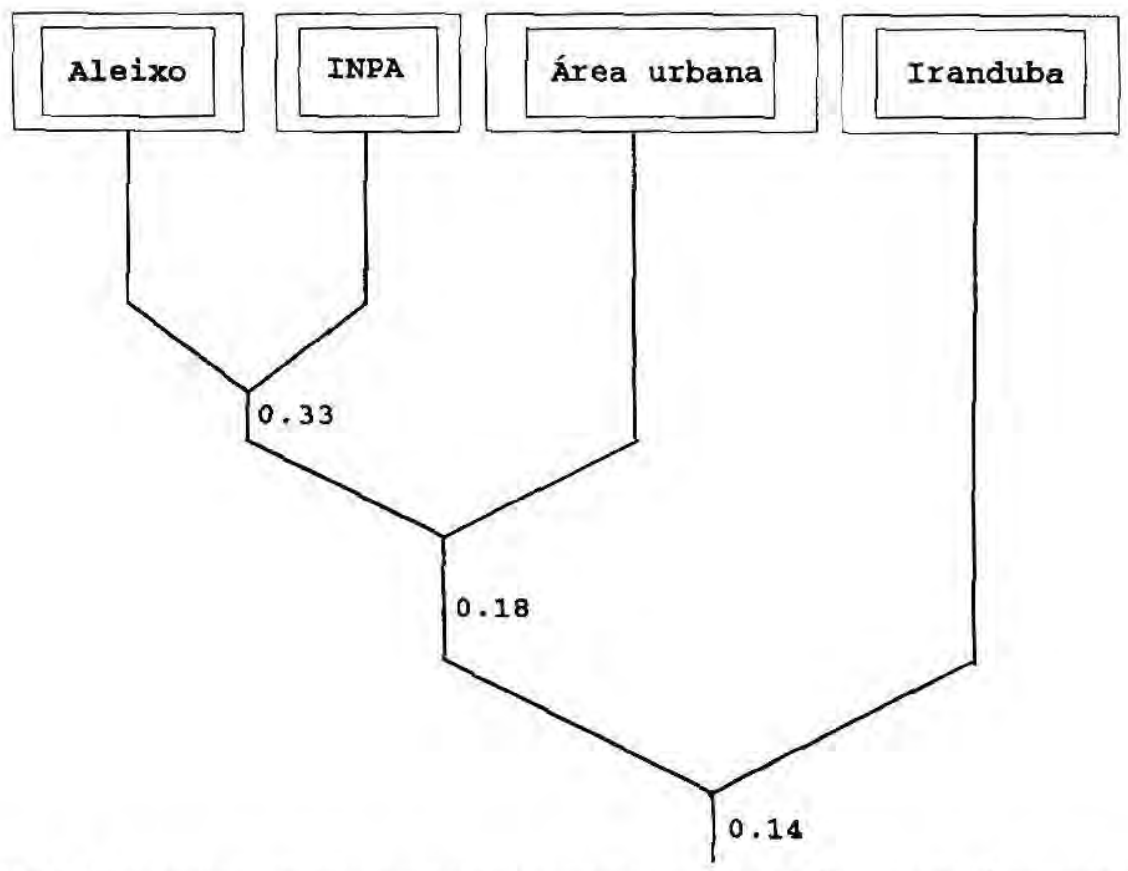

Figura 1. Dendrograma representativo das quatro comunidades, baseado no Quociente de Similaridade.

de $2478 \mathrm{~mm}$; temperatura média de $26,8^{\circ} \mathrm{C}$ e umidade relativa do ar média de $86 \%$. As características dos locais de coleta encontram-se em SILVA (1993).

\section{Análise faunística.}

Cada um dos quatro locais estudados foi considerado uma comunidade com características próprias. A caracterização de cada comunidade baseiou-se nos índices de freqüência, constância, abundância e dominância (SOUTHWOOD, 1966; SILVEIRA NETO et al., 1976). A delimitação da comunidade foi feita através do quociente de similaridade pela fórmula de Sorensen $\left(Q_{\mathrm{s} 1}=2 \mathrm{j} / \mathrm{a}+\mathrm{b}\right) \mathrm{e}$ o agrupamento através do desenvolvimento desses valores pela fórmula geral (SILVEIRA NETO et al., 1976),

$Q_{s 1} \frac{1}{m * n} \sum_{i=1 j=1}^{m} \sum_{s i}(A i * B j)$

\section{RESULTADOS E DISCUSSÃO}

Foram examinadas 205 amostras de frutos de 35 espécies botânicas. Foram obtidos parasitóides de larvas/pupas das moscas em 11 espécies de frutíferas (Tab. 1), correspondendo a 64 amostras. Emergiram 2630 parasitóides pertencentes a cinco espécies de Braconidae: Doryctobracon areolatus (Szépligeti, 1911), Opius bellus Gahan, 1930, Opius sp., Utetes (Bracanastrepha) anastrephae (Viereck, 1913), Asobara anastrephae (Muesebeck, 1958). Nos levantamentos foram coletados exemplares de dez espécies de Anastrepha (SILVA, 
Tabela 1. Número de frutos amostrados nas plantas hospedeiras de moscas-das-frutas das quais foram obtidos braconídeos.

\begin{tabular}{|c|c|c|c|c|}
\hline \multirow{2}{*}{ Hospedeiros } & \multicolumn{4}{|c|}{ COMUNIDADES } \\
\hline & Área urbana de Manaus & Aleixo & INPA & Iranduba \\
\hline Mapatí & - & - & 6040 & - \\
\hline \multicolumn{5}{|l|}{ Pouroma cecropiaefolia } \\
\hline Castanhola & 1955 & 738 & 33 & - \\
\hline \multicolumn{5}{|l|}{ Terminalia catappa } \\
\hline Bacuri & 224 & - & - & - \\
\hline \multicolumn{5}{|l|}{ Platonia insignis } \\
\hline Taperebá & 147 & 1496 & 344 & 1123 \\
\hline \multicolumn{5}{|l|}{ Spondias mombin } \\
\hline Gojaba & 36 & 12 & 201 & 2 \\
\hline \multicolumn{5}{|l|}{ Psidium guajava } \\
\hline Abiu & 16 & - & 18 & - \\
\hline \multicolumn{5}{|l|}{ Pouteria caimito } \\
\hline Manga & - & 168 & 27 & 23 \\
\hline \multicolumn{5}{|l|}{ Mangifera indica } \\
\hline Ingá-de-macaco & - & 803 & - & - \\
\hline \multicolumn{5}{|l|}{ Inga fagifolia } \\
\hline Mandioca* & - & 2 & 29 & 37 \\
\hline \multicolumn{5}{|l|}{ Manihot esculenta } \\
\hline Camu-camu & - & - & 4429 & 55 \\
\hline \multicolumn{5}{|l|}{ Myrciaria dubia } \\
\hline Mari & - & - & 519 & - \\
\hline Poraqueiba paraensis & & & & \\
\hline
\end{tabular}

* Refere-se ao número de brotos

1993). Entretanto, os braconídeos só puderam ser associados com oito espécies de Anastrepha (Tab. 2),

Opius sp. é morfologicamente muito similar em suas características a $O$. bellus, diferenciando-se desta, por apresentar as tíbias posteriores totalmente amareladas. $\mathrm{O}$ conhecimento taxonômico atual do gênero Opius não permite a identificação desses exemplares. De acordo com Wharton (inf. pes,), trata-se de uma espécie próxima de o. bellus. CANAL et al. (1994) consideraram os exemplares de
Alysiinae como Phaenocarpa anastrephae Muesebeck, 1958. Entretanto, Wharton (inf. pes.) considera esta espécie no gênero Asobara. Na realidade, a classificação do gênero dessa espécie tem sido discutida desde a sua descrição.

Das dez espécies de Braconidae relacionadas com moscas-das-frutas no Brasil (LEONEL JR. et al., n. publ.), cinco espécies ocorrem no Estado do Amazonas (LEONEL JR., 1991; SILVA et al., 1992; CANAL et al., 1994). 
Tabela 2. Número total de parasitóides nas respectivas frutíferas e espécies de moscas-das-frutas nas quatro comunidades.

\begin{tabular}{|c|c|c|c|c|c|c|c|c|}
\hline \multirow[b]{2}{*}{ Frutifera } & \multirow[b]{2}{*}{ Família } & \multirow[b]{2}{*}{$\begin{array}{l}\text { Espécies de } \\
\text { Anastrepha }\end{array}$} & \multirow[t]{2}{*}{ Parasitóides } & \multirow[t]{2}{*}{ D. areolatus } & \multirow[t]{2}{*}{ A. anastrephae } & \multirow[t]{2}{*}{ O. bellus } & \multirow[t]{2}{*}{ Opius sp. } & \multirow[t]{2}{*}{ U. anastrephae } \\
\hline & & & & & & & & \\
\hline $\begin{array}{l}\text { Psidium guajava } \\
\text { (Goiaba) }\end{array}$ & Myrtaceae & A. striata & $\cdot$ & 11 & - & - & - & - \\
\hline Spondias mombin & Anacardiaceae & Anastrepha spp.* & . & 29 & 35 & 50 & 2258 & 20 \\
\hline \multirow[t]{3}{*}{ (Taperebá) } & & A. obliqua & . & 8 & 1 & - & 1 & 1 \\
\hline & & A. antunesi & - & - & - & - & - & - \\
\hline & & A. bahiensis & . & - & - & - & - & - \\
\hline $\begin{array}{l}\text { Myrciaria dubia } \\
\text { (Camu-camu) }\end{array}$ & Myrtaceae & A. obliqua & - & 1 & - & - & - & - \\
\hline $\begin{array}{l}\text { Manihot esculenta } \\
\text { (Mandioca) }\end{array}$ & Euphorbiaceae & A. manihoti & . & - & - & - & - & 6 \\
\hline $\begin{array}{l}\text { Platonia insignis } \\
\text { (Bacuri) }\end{array}$ & Gutiferae & A. distincta & - & - & - & - & 1 & - \\
\hline Mangifera indica & Anacardiaceae & Anastrepha spp.* & - & 1 & - & - & 1 & 6 \\
\hline \multirow[t]{2}{*}{ (Manga) } & & A. obliqua & - & - & - & - & - & 12 \\
\hline & & A. fraterculus & - & - & - & - & - & - \\
\hline Terminalia catappa & Combretaceae & Anastrepha ssp. * & - & 14 & - & - & - & - \\
\hline \multirow[t]{2}{*}{ (Castanhola) } & & A. fraterculus & - & 1 & - & - & - & - \\
\hline & & A. obliqua & - & . & - & - & - & - \\
\hline Pouteria caimito & Sapotaceae & A. leptozona & $\therefore$ & 16 & - & - & - & - \\
\hline (Abiu) & & & - & - & - & - & - & - \\
\hline Pouroma cecropiaefolia & Moraceae & Anastrepha $s p p^{*}$ & - & 96 & 6 & - & 2 & 30 \\
\hline (Mapati) & & A. bahiensis & - & 19 & - & - & - & - \\
\hline Poraqueiba paraensis & Icacinaceae & A. leptozona & - & - & - & - & 1 & - \\
\hline (Mari) & & & . & - & - & - & - & - \\
\hline Inga fagifolia & Fabaceae & A. distincta & - & - & - & - & 1 & - \\
\hline (Ingá-de-macaco) & & & - & - & - & - & - & - \\
\hline
\end{tabular}

* Năo fol possivelassociar a espécie de mosca com a do parasitóide 
A maioria dos parasitóides foi obtida de larvas/pupas das moscas em frutos de taperebá (Spondias mombin L., Anacardiaceae), evidenciando a influência do fruto no parasitismo. Este fato é realçado por Opius sp., que emergiu em maior quantidade de frutos de taperebá, apesar das mesmas espécies de Anastrepha estarem presentes também em outras espécies de frutíferas (Tab. 2). NISHIDA \& NAPOMPETH (1974) observaram maior atratividade do parasitóide pelo tecido da fruta do que propriamente pela larva da mosca. BRESSAN (1987), ao comparar o parasitismo de moscas-das-frutas em seriguela (Spondias purpurea L.) e carambola (Averrhoa carambola L., Oxalidaceae), observou que é maior a possibilidade do parasitóide achar a larva da mosca em frutos com pouco volume (no caso seriguela). LEONEL JR. (1991), amostrando frutos de 20 espécies de frutíferas, obteve maiores níveis de parasitismo nos frutos de casca fina e polpa rasa. MESSING \& WONG (1992) evidenciaram a influência do odor do fruto sobre a atração do parasitóide.

Apenas em Iranduba, foram coletados exemplares das cinco espécies de parasitóides. No bairro distrital do Aleixo, a supremacia de Opius sp. sobre as demais espécies foi marcante. Também, o número de espécimens de Opius sp. obtido nesse local foi muito mais alto do que nas outras comunidades. Do total de 2630 parasitóides coletados, 2080 exemplares de Opius sp. (79,08\%) foram obtidos das larva/pupas das moscas que infestavam principalmente taperebá, no Aleixo (Tab. 3).
É preciso ressaltar que o taperebá, também ocorre nas outras comunidades estudadas. A diferença no número de parasitóides coletados, nessa planta hospedeira em cada comunidade, não deve ser atribuída ao número de frutos amostrados. No Aleixo, foram colhidos 1496 frutos, enquanto que em Iranduba, onde obteve-se o menor número de parasitóides, foram colhidos 1123 frutos dessa planta hospedeira.

De acordo com WONG et al . (1984), STARCK et al. (1991) e VARGAS et al. (1993), as populações de parasitóides são diretamente influenciadas pelas populações do tefritídeo hospedeiro. Assim, o elevado nível populacional de Opius sp. no Aleixo poderia estar relacionado à maior população de moscas-das-frutas nesse local, comprovada pela grande quantidade de pupas de moscas obtidas, principalmente do taperebá. Entretanto, a quantidade de pupas de moscas-das-frutas foi grande também na Estação Experimental do INPA, mas a população de parasitóides foi pequena (Tab. 3). Portanto, outros fatores, além do tefritídeo hospedeiro, devem influir no nível populacional dos braconídeos nos locais estudados.

\section{Análise faunística por local.}

$\mathrm{Na}$ área urbana de Manaus, foram coletadas quatro espécies de braconídeos. Opius sp. foi mais abundante e dominante. É também a única espécie constante nas amostras e representou $73,82 \%$ dos exemplares coletados. $D$. areolatus e $U$. anastrephae 
Tabela 3. Análise faunística dos parasitóides coletados nas quatro comunidades.

Espécie
Manaus(área urbana)

Manaus (INPA)

( Manaus (A)

N F C A D

D. areolatus

4

2.10 y $<$ n

$\begin{array}{lllll}N & F & C & A & D\end{array}$

o. bellus

$32 \quad 16.75$ z $c$ n

U. anastrephae

14

7.33 y $0 \mathrm{n}$

37

16.59 z $c n$

141

73.82 y $c$ c $n$

14

6.28 z $c$ n

$35 \quad 15.70 \quad z \quad c \quad n$

\begin{tabular}{lllll}
\multicolumn{4}{c}{ Manaus (Aleixo) } \\
\hline N & F & C & A & D
\end{tabular}

\begin{tabular}{ccccc}
\multicolumn{4}{c}{ Iranduba } \\
\hline N & F & C & A & D \\
\hline 16 & 24.80 & $w$ & C & S
\end{tabular}

A. anastrephae

223

41

1.90 w c n

16

24.80

12

$0.56 \times c n$

6

9.68 z $<~ n$

21

0.97 y $c \quad n$

3

4.84 y c $n$

2080

$96.56 \mathrm{w}$ ma s

30

48.39 y $c$ n

Toatal parasitóides 191

223

2154

$\begin{array}{lllll}7 & 11.29 & \text { y } & \text { c } & n\end{array}$

$\%$

7.26

8.48

81.90

2.4

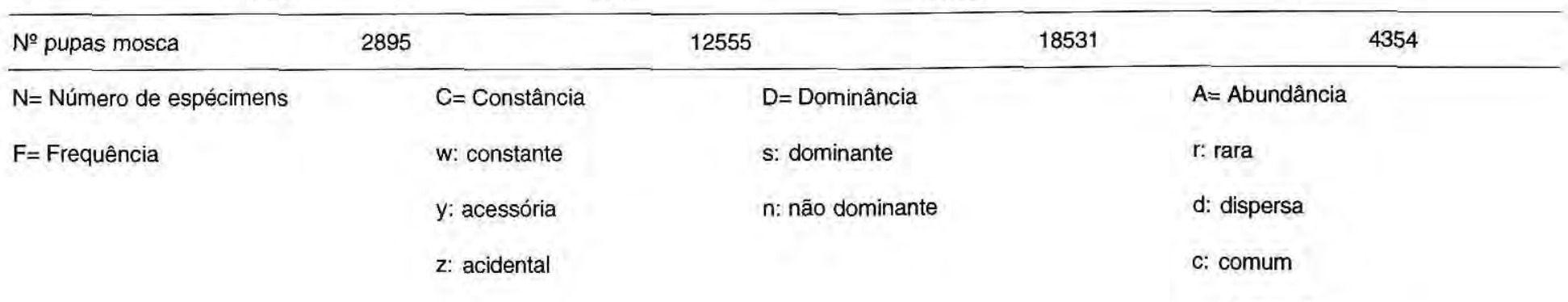

a: abundante

ma: muito abundante 
apresentaram frequências de $2,10 \%$ e $7,33 \%$, respectivamente, mas são espécies acessórias nas coletas e comuns na região, ou seja, estão presentes na área toda, mas não o tempo todo. $O$. bellus foi coletada em poucas amostras, mas com maior número de exemplares por amostra, assim, apresenta maior freqüência $(16,75 \%)$, sendo acidental nas coletas.

$\mathrm{Na}$ estação experimental do INPA, quatro espécies foram coletadas. D. areolatus é dominante, mais abundante, mais freqüente e também a mais constantemente coletada. As outras três espécies da região foram $U$, anastrephae, Opius sp. e A. anastrephae. Todas as três são acidentais nas coletas, mas comuns na região. Uma espécie acidental e comum indica uma população distribuída na-região, mas afetada por algum fator que determina o seu aparecimento em alguns períodos ou locais, como por exemplo, a população do seu hospedeiro.

No bairro distrital do Aleixo, em Manaus, devido à proximidade com a área urbana, foram coletadas as mesmas espécies de parasitóides, que apresentaram índices faunísticos semelhantes. Assim, Opius sp. foi a espécie dominante, muito abundante $\mathrm{e}$ constante nas coletas e frequiência na área de $96,56 \%$. As outras quatro espécies foram pouco freqüentes: $D$. areolatus $(1,90 \%), O$. bellus $(0,56 \%), U$. anastrephae $(0,97 \%)$. Apesar da baixa frequêencia, $D$. areolatus foi constante nas amostras e é comum na área. Suas populações sempre estão presentes na região, mas com poucos indivíduos. $U$. anastrephae foi comum e acessória.
Em Iranduba, coletaram-se cinco espécies, todas comuns quanto a abundância. D. areolatus e Opius sp. são as espécies dominantes na área. Opius sp. é a mais freqüente $(48,39 \%)$ e $D$. areolatus a mais constantemente coletada. A. anastrephae e $U$. anastrephae são acessórias, com freqüências de $11,29 \%$ e $4,84 \%$, respectivamente. $O$. bellus foi acidental e com freqüência de $9,68 \%$.

\section{Análise faunística geral.}

A espécie predominante foi Opius sp., que apresentou as maiores frequiências, foi dominante em três áreas e abundante em duas. Esta espécie foi constante nas áreas urbanizadas (Aleixo e centro de Manaus) e acidental na área rural (INPA).

$D$. areolatus destacou-se entre todas por ser a mais constante nas coletas. Devese salientar que esta espécie apresentou maiores frequiências na área rural, que diminuem para a área urbanizada. A espécie foi predominante nas áreas rurais, onde existiu maior diversidade de espécies de Anastrepha e de frutíferas. O fato dessa espécie se apresentar constantemente nas coletas pode indicar baixa especificidade em relação ao hospedeiro.

A terceira espécie, que ocorreu nos quatro locais, foi $U$. anastrephae, todavia, com baixa freqüência, nunca dominante $\mathrm{e}$ sempre comum e acidental ou acessória. $O$. bellus ocorreu na área urbanizada, onde teve maior frequiência. Foi acidental nas coletas, indicando provavelmente maior especificidade, $A$, anastrephae apresentouse somente na área rural, sendo espécie acidental nas áreas amostradas. 
A preferência dos braconídeos por determinado local já tinha sido observada por vários autores. NISHIDA (1955) encontrou menor parasitismo de Opius fletcheri Silvestri, 1916 numa plantação do que numa área não cultivada. VARGAS et al. (1993) observaram Biosteres arisanus (Sonan, 1932) num pomar de frutíferas e $B$. vandenboshi (Fullaway, 1920) numa mata não cultivada, ao lado do pomar. ARRIGONI (1984) coletou quatro espécies de parasitóides de larvas/pupas de moscas-das-frutas numa área com diversas espécies de frutíferas, em Limeira (SP), e nenhuma espécie foi obtida numa área com predominância de frutas cítricas em Piracicaba (SP). Aquele autor obteve mais parasitóides em frutos de Myrtaceae, sendo $D$. areolatus mais comum. Esse fato poderia estar associado à preferência de Anastrepha por frutos de Myrtaceae (MALAVASI et al., 1980).

Nos locais estudados, as espécies de Anastrepha também infestam frutos de várias espécies de Myrtaceae (SILVA, 1993), no entanto, a coleta de braconídeos nessa família de hospedeiros foi insignificante (Tab. 2). Em realidade, os frutos de Anacardiaceae exerceram maior atração sobre os braconídeos nos locais amostrados. Portanto, outros fatores,
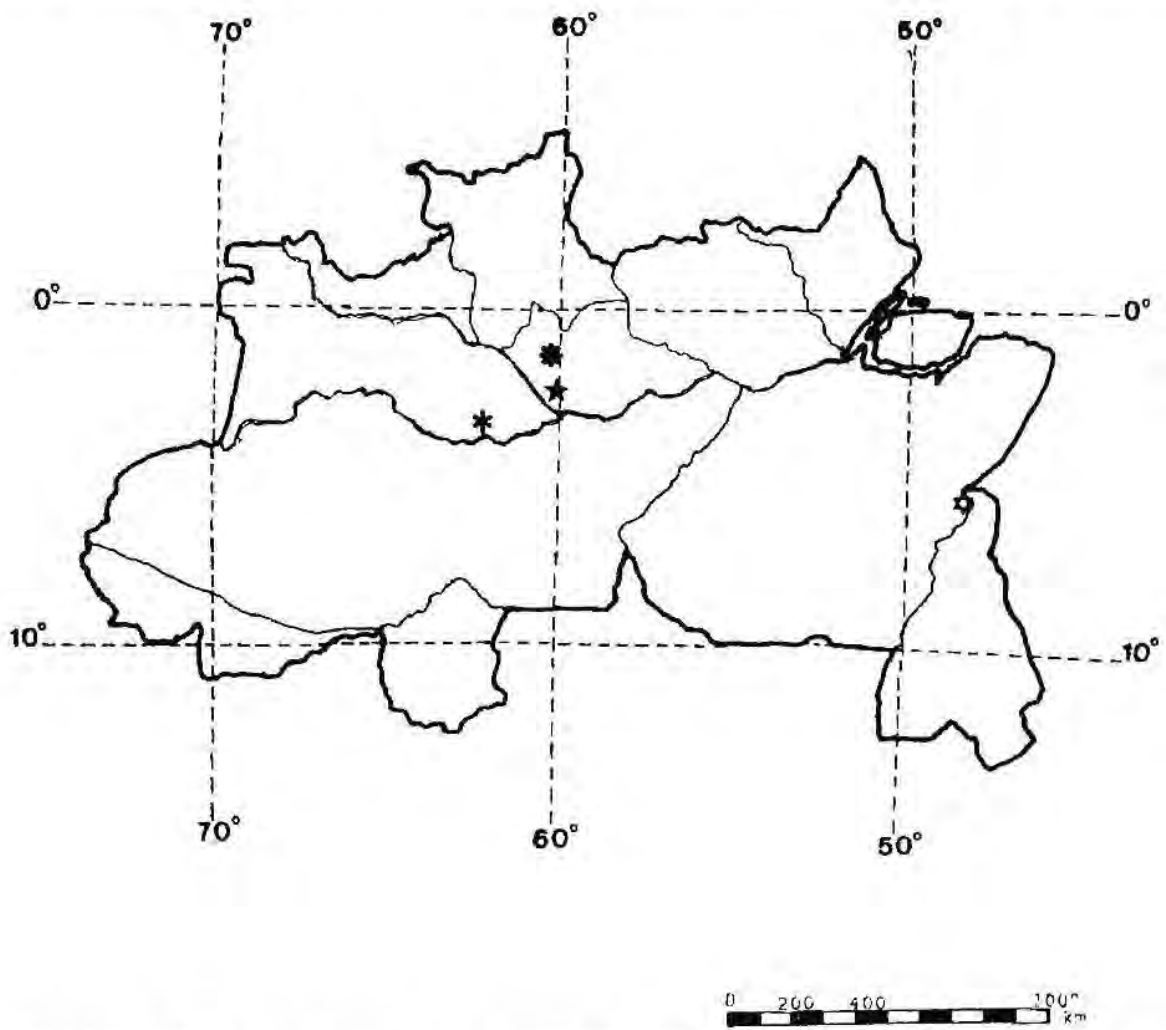

Figura 2. Locais de coleta dos parasitóides de mosca-das-frutas no Estado do Amazonasm ( $\star$ zona urbana de Manaus e Aleixo; INPA/BR - 174 km 40; * Ianduba). 
além dos tefritídeos hospedeiros (discutido anteriormente) e das plantas hospedeiras, têm influência sobre a ocorrência dos braconídeos em determinada área.

\section{Delimitação das comunidades.}

Os fatores anteriormente discutidos influenciaram a diversidade de parasitóides nas diferentes comunidades. Assim, com relação à composição de espécies, a área urbana de Manaus e o Aleixo são regiões com $100 \%$ de semelhança; Iranduba apresentou $89 \%$ de similaridade em relação a esses dois locais e o INPA (BR-174 km 40) foi o local que mais diferiu das demais comunidades, com índice de similaridade de $82 \%$ (Fig. 2). De fato, na área urbana de Manaus e no Aleixo ocorreram as mesmas espécies de parasitóides, que apresentaram os mesmos índices faunísticos. Esses resultados podem ser devidos ao fato desses dois locais situarem-se numa mesma área urbana. Iranduba e o INPA são áreas com maior influência da floresta, porém com alguma diferenciação fisiográfica. Assim, Iranduba é área de várzea, enquanto a estação do INPA localiza-se em terrafirme e as coletas, nesse local, foram feitas em pomares experimentais. Esses resultados coincidem com os obtidos por SILVA (1993), que analisou, através da Análise de Agrupamentos, essas quatro comunidades em relação às populações de espécies de Anastrepha e suas plantas hospedeiras.

\section{Agradecimentos}

Os autores agradecem ao Dr.
Robert A. Wharton (Texas A\&M University, College Station, USA) pelas informações sobre os parasitóides.

\section{Bibliografia Citada}

ARRIGONI, E.B. 1984. Dinâmica populacional de moscas-das-frutas (Diptera: Tephritidae) em três regiões do Estado de São Paulo. Tese de Doutoramento, Escola Superior de Agricultura "Luiz de Queiroz", Universidade de São Paulo, Piracicaba, São Paulo, 166 p.

BRESSAN, S. 1987. Aspectos do comportamento reprodutivo e ecológico de Anastrepha obliqua (Macquart,1835) (Diptera, Tephritidae) na natureza. Tese de Doutoramento, Faculdade de Medicina de Ribeirão Preto, Universidade de São Paulo, Ribeirão Preto, São Paulo, $139 \mathrm{p}$.

CANAL D., N.A.; ZUCCHI, R.A.; SILVA, N.M.; LEONEL JR., F.L. 1994. Reconocimiento de las espécies de parasitóides de (Hym: Braconidae) de moscas de las frutas (Dip.: Tephritidae) en dos municípios del Estado de Amazonas, Brasil. Boletin del Museo de Entomologia de la Universidad del Valle, 2(1,2):1-17.

CLAUSEN, C.P. 1956. Biological control of fruit flies. Journal of Economic Entomology, 49(2):176-178.

GAULD, I; BOLTON, B. 1988. The Hymenoptera. London, British Museum; Oxford University Press, 332 p.

LEONEL JR., F.L. 1991. Espécies de Braconidae (Hymenoptera) parasitóides de moscas-dasfrutas (Diptera: Tephritidae) no Brasil. Tese de Mestrado, Escola Superior de Agricultura "Luiz de Queiroz", Universidade de São Paulo, Piracicaba, São Paulo, 83 p.

LEONEL JR., F.L.; ZUCCHI, R.A.; WHARTON, R.A. (n. publ.), Distribution and tephritid hosts (Diptera) of braconid parasitoids ( $\mathrm{Hy}$ menoptera) in Brazil.

MALAVASI, A.; MORGANTE, J.S.; ZUCCHI, R.A. 1980. Biologia de moscas-das-frutas (Diptera, Tephritidae). I. Lista de hospedeiros e ocorrência. Revista 
Brasileira de Biologia, 40(1):9-16.

MESSING, R.H. ; WONG, T.T.Y. 1992. An effective trapping method for field studies of opiine braconid parasitoids of tephritid fruit flies. Entomophaga, 37(3): 391-396.

NISHIDA, T. 1955. Natural enemies of the melon fly, Dacus cucurbitae Coq. in Hawaii. Annals of the Entomological Society of America, 48(3):171-177.

NISHIDA, T; NAPOMPETH, B. 1974. Trap for tephritid fruit fly parasites. Entomophaga, 19(3):349-352.

SILVA, N.M. 1993. Levantamento e análise faunistica de moscas-das-frutas (Diptera, Tephritidae) em quatro locais do Estado do Amazonas. Tese de Doutoramento, Escola Superior de Agricultura "Luiz de Queiroz", Universidade de São Paulo, Piracicaba, São Paulo, 154 p.

SILVA, N.M.; LEONEL JR., F.L.; ZUCCHI, R.A.; SILVEIRA NETO, S. 1992. Levantamento de Braconidae ( $\mathrm{Hy}$ menoptera), parasitóides de moscas-dasfrutas (Dip.: Tephritidae) em dois municípios do Estado do Amazonas. In: Anais do $3^{\circ}$ Simpósio de Controle Biológico, EMBRAPA/CNPDA, Jaguariuna, p. 224.

SILVEIRA NETO, S.; NAKANO, O.; BARBIN, D.; VILLA NOVA, N.A. 1976. Manual de ecologia dos insetos. São Paulo, Editora Agronômica Ceres. 420 p.
SOUTHWOOD, T.R.E. 1966. Ecological methods. London, Methuen \& Co. Ltd. 391 p.

STARK, J.D.; VARGAS, R.I; THALMAN, R.K. 1991. Diversity and abundance of oriental fruit fly parasitoids (Hymenoptera: Braconidae) in guava orchards in Kauai, Hawaii. Journal of Economic Entomology, 84(5):1460-1467.

VARGAS, R.I;; STARK, J.D.; UCHIDA, G.K.; PUCERLL, M. 1993. Opiinae parasitoids (Hymenoptera: Braconidae) of oriental fruit fly (Diptera: Tephritidae) on Kauai Island, Hawaii: Island wide relative abundance and parasitism rates in wild and orchard guava habitats. Environmental Entomology, 22(1):246-253.

WHARTON, R.A. 1989. Classical biological control of fruit-infesting Tephritidae. In: ROBINSON, A.S. \& HOOPER, G., ed. Fruit flies; their biology, natural enemies and control. Elsevier, New York, v.2, p.303-313. (World Crop Pests, 3B).

WHARTON, R.A. 1993. Bionomics of the Braconidae. Annual Review of Entomology, 38:121-143.

WONG, T.T.Y.; MOCHIZUKI, N.; NISHIMOTO, J.I. 1984. Seasonal abundance of parasitoids of the mediterranean and oriental fruit flies (Diptera: Tephritidae) in the Kula area of Mavi, Hawaii. Environmental Entomology, 13(1):140-145. 